\title{
El derecho a la ciudad. Una mirada desde América Latina*
}

The right to the city. A view from Latin America

Ester Schiavo Alejandro Gelfuso

Paula Vera

\section{Resumen}

El propósito de este artículo es realizar un aporte a la configuración de una perspectiva latinoamericana respecto del "derecho a la ciudad". El pensamiento dominante, sostenido en las epistemologías del norte, ha obturado durante cierto tiempo la búsqueda de una perspectiva crítica propia, es decir, del sur, que contribuya a agudizar una mirada alternativa. Para ello se indagan dos tensiones. Por un lado, la existente entre el modelo de sociedad propuesto e implementado por el neoliberalismo frente al impulsado en ciertos países de la región y con distintos matices por el post-neoliberalismo. Y, por otro lado, la disputa respecto al significado atribuido al derecho a la ciudad.

Palabras clave: derecho a la ciudad; América Latina; perspectiva crítica; neoliberalismo; post-neoliberalismo.

\begin{abstract}
The purpose of this article is to make a contribution to the establishment of a Latin American perspective on the "right to the city ". The dominant thought, held in the northern epistemologies, has blocked, during a certain time, the search for a southern critical perspective that can help to sharpen an alternative view. Therefore, two tensions are investigated. On the one hand, the tension that exists between the model of society proposed and implemented by neoliberalism, in opposition to the model stimulated in certain countries of the region, with different shades, by post-neoliberalism. And, on the other hand, the dispute with regard to the meaning attributed to the right to the city.
\end{abstract}

Keywords: right to the city; Latin America; critical perspective; neoliberalism; post-neoliberalism. 


\section{El derecho a la ciudad entre el capitalismo actual y la apropiación de su significado}

El presente trabajo tiene como objetivo analizar las significaciones actuales del denominado "derecho a la ciudad" desde una mirada latinoamericana. A tal fin, se plantea indagar los aportes de dicha propuesta al pensamiento crítico sobre la problemática urbana, como una iniciativa autónoma del pensamiento hegemónico.

Desde esta perspectiva, cabe preguntarse por el itinerario teórico-práctico de dicho concepto en un continente signado por la acelerada urbanización, la desigualdad y la expulsión social, la fragmentación espacial y los conflictos territoriales. Se hace referencia tanto a aquellos procesos ocurridos en las décadas signadas por la aplicación a ultranza de políticas neoliberales, fuertemente materializadas en las tramas urbanas latinoamericanas; como a las transformaciones económicas, políticas y culturales ocurridas a partir de un nuevo periodo de crecimiento tras las sucesivas crisis que acontecieron en los países de la región, etapa a la cual, para diferenciarla de la anterior, siguiendo a Sader (2008) y García Linera (2012), se la denomina post-neoliberalismo.

En dicho contexto, para pensar el derecho a la ciudad desde una perspectiva latinoamericana, se plantea avanzar en el marco de dos tensiones principales. En primera instancia, se propone situarse en la tensión existente entre dos modelos de sociedad antagónicos, el propuesto e implementado por el neoliberalismo particularmente en la década del 90, frente al impulsado en los últimos años en ciertos países de la región y con distintos matices por el post-neoliberalismo. No obstante, como en todo proceso histórico, no se trata de un punto de inflexión, de un antes y un después, sino que es evidente que existen continuidades y discontinuidades entre una y otra etapa. Así, a pesar de la autonomía relativa entre política y mercado que caracteriza a la última década en Latinoamérica, las dificultades de los gobiernos post-neoliberales para proyectar territorialmente sus políticas en las ciudades tienen relación con el desarrollo alcanzado por los procesos de neoliberalización remanentes (Theodore, Brennet y Peck, 2009), cuyas iniciativas continúan vigentes e imponen límites claros a la hora de proponer transformaciones profundas relacionadas, por caso, tanto con el acceso a determinadas plataformas materiales como con la participación de la ciudadanía en los procesos de decisión.

En segunda instancia, interesa profundizar sobre la tensión existente respecto al significado atribuido al derecho a la ciudad; dado que para algunos gobiernos de la región el mismo parece reducirse, o limitarse, a una apropiación normativa-institucional. Es decir, para los nuevos gobiernos que se consolidaron tras la crisis de legitimidad política-estatal del neoliberalismo, el derecho a la ciudad parece haber sido movilizado para contener una problemática urbana que las reformas institucionales democráticas no han podido atender, pero mostrando incapacidad para iniciar el tipo de metamorfosis radical proyectada por Lefebvre (1969) en su obra homónima. Así, el derecho a la ciudad se 
presenta como un significante vacío (Laclau, 2005), un nodo discursivo que permite conjugar demandas particulares dispersas, disputado por diversos actores que proyectan en dicho concepto su modelo de sociedad. Una relación conflictiva entre quienes producen y mejoran los bienes comunes urbanos en diversas escalas y quienes se apropian de ellos (Harvey, 2013).

El trabajo se enfoca principalmente en estas dos tensiones, abordándolas mediante un itinerario que propicie la valoración tanto de las elaboraciones académicas, como las prácticas de los sujetos emergentes de los modelos en disputa. Siguiendo a Boaventura de Sousa Santos (2011) asistimos a una disonancia entre la teoría crítica socio-política y las prácticas. Mientras la mayor parte de la producción teórica se desarrolla en el norte global, las iniciativas colectivas novedosas y transformadoras (tanto organizadas como espontáneas) están ocurriendo en el sur global. Este factor profundiza aún más la necesidad de construir las epistemologías del sur, un pensamiento alternativo de alternativas. En este marco se pretende posicionar al derecho a la ciudad en América Latina y de este modo, analizar la situación de las ciudades latinoamericanas y su paso de escenarios a objetos de disputa.

\section{Neoliberalización o neoliberalismo realmente existente en las ciudades latinoamericanas}

Para abordar las complejidades de las ciudades latinoamericanas del siglo XXI se propone ampliar el análisis más allá del neoliberalismo a secas, dando lugar a una lectura procesual que sitúe una parte del escenario de disputa bajo el concepto de neoliberalización o neoliberalismo realmente existente, acuñado por Theodore, Brennet y Peck (2009), y neoliberalismo desde abajo utilizado por Gago (2014). En principio, porque dichas miradas permiten un acercamiento al neoliberalismo desde la óptica de la materialización, la inserción contextual de diversos proyectos de reestructuración, su proyección al interior de contextos nacionales, regionales $y$, principalmente, locales. Lo "realmente existente" hace referencia a la especificidad de los marcos institucionales, políticos, regulatorios y culturales donde deben hacerse realidad dichas políticas. El neoliberalismo desde abajo, por su parte, se plantea como un conjunto de saberes, tecnologías y prácticas que despliegan una racionalidad de nuevo tipo, es decir, una forma anclada en los territorios, fortalecida en las subjetividades populares, y expansiva y proliferante en términos organizativos en las economías formales $y$, sobre todo, informales.

Por otra parte, durante esa etapa, las competencias de los gobiernos locales y metropolitanos, cuando los hay, en relación con los estados-nación, han sido una de las formas significativas en las que una escala particular de organización humana ha resignificado su papel. Las escalas jerárquicas en las que se organizan hoy los espacios urbanos son diferentes a las de hace 30 años. Los neoliberalismos realmente existentes son coyunturalmente específicos, porque la neoliberalización, más que una fase, o un sistema estable y autónomo, es un proceso 
abierto compuesto por diversas estrategias de reestructuración que se interceptan de distintos modos.

En las ciudades latinoamericanas, el programa concreto de materialización y reestructuración neoliberal combina, por un lado, el desmantelamiento de formas institucionales estatales que le son ajenas (como los sistemas redistribucionistas y colectivos) desregulando la economía al servicio de intereses corporativos; y por otro, el lanzamiento de nuevas modalidades de regulación institucional y nuevas formas de gestión estatal.

En la región, el proceso de neoliberalización fue, siguiendo a de García Linera (2012), un sofisticado mecanismo con implicancias políticas y culturales para expropiar riquezas y bienes comunes colectivos mediante la privatización de lo público estatal - convertido ahora en privado local o extranjero - y lo público no estatal, es decir, el conjunto de bienes comunes que, como bien señala su nombre, no son del estado sino del común de los habitantes. A medida que la política neoliberal reduce la financiación de bienes públicos, también mengua el bien común disponible. La educación, la vivienda, el transporte, las dificultades para el acceso a determinadas plataformas de productividad y de cultura, por caso, son rasgos habituales que aún prevalecen como síntoma estructural a pesar de haberse atemperado en los últimos años.

Las ciudades latinoamericanas fueron blancos geográficos y laboratorios institucionales para experimentar formas de neoliberalización tales como la creación de zonas empresariales, reducción de impuestos locales e impulso masivo de diversas formas de asociación público-privada, entre otras. Un conjunto de procesos que acondiciona espacios de la ciudad tanto para el crecimiento económico como para el hiper-consumo.

Así, los gobiernos locales encuentran en la actividad inmobiliaria, casi siempre muy visible, un aporte de legitimidad en términos de las ideologías en boga de la modernización urbana, la formación de ciudades globales, o la inserción en la globalización (Pradilla Cobos, 2013), aunque sin definir específicamente a qué se hace referencia cuando se enuncian dichos conceptos. Esto explica, en parte, porqué buena parte de los gobiernos locales (independientemente de su orientación política) se ajustaron a visiones de corto plazo propias de la competitividad, el marketing territorial y las desregulaciones para atraer inversiones, aun a pesar de que este fortalecimiento de las ciudades como protagonistas dentro de un esquema global que atrae flujos y materializaciones, tiene viabilidad en un número relativamente reducido de las mismas.

¿Cuáles son las transformaciones mediante las que se materializa la neoliberalización de las ciudades latinoamericanas? ¿Cómo se produce el espacio urbano durante este proceso? Cintia Baremboin (2010) destaca tres características. La primera es la preocupación por parte de los sectores empresariales por estimular el papel del mercado y del capital privado en el desarrollo de la configuración de las ciudades mediante la conflictiva - "pero necesaria"construcción (espacial) de sociedades público-privadas. Se consolida así la utopía de los promotores inmobiliarios (Harvey, 2012), donde cada nueva oleada de inversión pública 
es orientada por los intereses privados, creando un esquema donde el primero asume los riesgos y el segundo absorbe los beneficios, a la vez que reinvierte su excedente de capital en el proceso de urbanización. La segunda característica es la transformación del rol estatal mediante la descentralización y la profundización de las políticas locales de gobierno, que incluyen entre sus funciones la conducción de una variable económica tan importante como la urbanización. Finalmente, para esta autora, la tercera característica, alude a una serie de fenómenos que requieren un análisis algo más profundo. Se trata del desarrollo conflictivo y dinámico de una nueva geografía urbana producto de las nuevas modalidades de crecimiento y consumo. Las ciudades modifican los modos de expansión de sus fronteras y su estructura económica. Surgen, de forma aparentemente inconexa, nuevas "centralidades" que encarnan los artefactos del consumo, como malls, complejos empresariales, barrios cerrados, y se multiplican las zonas anexas mediante una descontrolada expansión horizontal, con escaso uso sustentable de suelo y subvenciones públicas para las redes de infraestructura de transporte, luz, gas, provisión de agua y desagües, entre otras, todo lo cual favorece principalmente a ciudadanos de rentas elevadas y a las empresas particulares que gestionan dichos proyectos urbanos.

Es evidente que la expansión terciaria que sustituye a la industrial se ha manifestado en la morfología estructural urbana (Pradilla Cobos, 2013) tanto en su polo formal como informal. Lo que posibilita la convivencia, no menos conflictiva, entre núcleos reducidos, altamente tecnificados con circulación de mercancía, régimen laboral y salarial, y alrededor de ello una economía informal, flexible y precaria, sin regulación ni derechos, de la cual emerge lo que Harvey (2013) Ilama precariado, en clara alusión a la desterritorialización del trabajo. De dicho polo informal surge uno de los eslabones débiles del proceso de neoliberalización y también del relato post-neoliberal: la crisis de violencia urbana. En palabras de Gorelik (2004), aquella representación latinoamericanista surgida como proyecto en los sesenta y setenta se realizó en los años 90, aunque ya no como proyecto sino como destino: la inseguridad, el blindaje privado y la extinción del espacio público.

\section{Post-neoliberalismo realmente existente en las ciudades latinoamericanas}

A partir del análisis de cada una de estas transformaciones, se puede afirmar que el prefijo Post, en el vocablo post-neoliberalismo, no indica ni transición ni mera superación, sino más bien la crisis de legitimidad del patrón neoliberal, su antecesor, como política estatal-institucional a partir de una diversidad de revueltas sociales ¿Qué tipo de política protagoniza el post-neoliberalismo y qué economías sustenta? Por último ¿qué uso del espacio urbano hace?

Primero y principal, se presenta como el fortalecimiento del Estado en contraposición a una lógica, previamente experimentada en todo el continente, de subordinación de la propiedad y riqueza estatal a la acumulación privada. 
La intervención regulatoria para garantizar transferencias de recursos financieros al ámbito productivo es un ejemplo transversal en un importante número de países de América Latina durante la última década.

Siguiendo en la misma senda, la segunda característica se refiere a los procesos de nacionalización de determinados recursos públicos, para convertirlos en empresas del Estado, que remite, al menos en sus formas, al estado de bienestar que funcionó como articulador social compensando desigualdades con una intervención activa en la economía y el funcionamiento cotidiano de la sociedad mediante políticas con sesgo universal. La revalorización de la propiedad pública estatal se convierte en un factor central para definir el posicionamiento ante los problemas estructurales de las sociedades latinoamericanas. Es decir, la soberanía ligada a la capacidad material y ésta, a la propiedad pública estatal. Si el neoliberalismo se caracterizó por la externalización del excedente, el post-neoliberalismo lo internaliza y direcciona hacia el consumo interno con el objetivo de ampliar los recursos comunes reforzando la posibilidad de darles un fin colectivo. Los procesos de nacionalización y de recuperación del control regulador de la economía se asocian al imaginario político de un estado garante de determinados derechos básicos, donde los recursos son centrales.

Una tercera característica que presentan los gobiernos post-neoliberales es la desmercantilización selectiva de la economía, mediante la intervención del Estado en ciertas áreas y actividades básicas introduciendo no una lógica de mercado, valor de cambio, sino una lógica de valor de uso. Una concepción de suma importancia tanto en ámbitos urbanos como rurales, porque permite, al menos como horizonte posible, proyectar un número creciente de actividades de la vida cotidiana cuya satisfacción no tiene porqué moverse necesariamente dentro de las leyes del mercado.

Como se sostiene desde el comienzo de este trabajo, dichos enunciados hacen pie en un escenario en tensión permanente con los procesos de neoliberalización. Un ejemplo visible es el mercado inmobiliario urbano, su lógica como bien de cambio y su influencia en los procesos de urbanización, que ha crecido notablemente en casi la totalidad de las ciudades latinoamericanas a pesar de la recuperación e instalación del discurso en favor de la soberanía pública estatal en áreas centrales de la economía (hidrocarburos, petróleo, agua, luz, aerolíneas, entre otros).

En relación a las ciudades, la nueva dinámica espacial producto de la reestructuración económica y del empleo, el impacto de las nuevas tecnologías, la reforma del Estado y la descentralización, los cambios culturales y nuevas pautas de consumo y movilidad, modifican también los patrones centrales de los procesos de urbanización, profundizando su perspectiva económica en detrimento de la política y social. La inédita etapa de crecimiento económico y aumento del consumo que atravesó América Latina en la última década, desde la metafórica mirada de Natanson (2014), aportó al crecimiento puertas adentro, pero no puertas afuera. El inicio de la segunda década del siglo XXI presenta acontecimientos socio-políticos complejos, en el que las metrópolis latinoamericanas son protagonistas centrales y en cuyo territorio se 
configura una nueva trama de conflictividad donde coexisten, por un lado, la vitalidad de un proceso de crecimiento que atenuó los reclamos básicos de alimentación y empleo, y por otro, las limitaciones de las respuestas a nuevas demandas por parte de las distintas dependencias del estado. Aun con cambios importantes en las estructuras de gobierno a nivel continental, la neoliberalización se ha extendido por el mundo como una vasta marea de reforma institucional y ajuste discursivo.

Aunque han ocurrido cambios en las orientaciones en las políticas territoriales, no se han construido patrones tendenciales de cambios territoriales, urbanos en particular (Pradilla, 2013). Entonces, es este un marco pertinente para preguntarse por el derecho a la ciudad para aportar al pensamiento crítico latinoamericano sobre la problemática urbana, como una iniciativa autónoma del pensamiento hegemónico.

\section{El derecho a la ciudad y sus apropiaciones antagónicas en América Latina}

Como se afirma al comienzo de este trabajo, interesa profundizar la tensión existente respecto al significado atribuido al derecho a la ciudad; dado que para algunos gobiernos de la región el mismo parece reducirse a la apropiación normativa-institucional, lo cual resulta antagónico, o al menos no funcional, frente a la propuesta por Lefebvre.

La apropiación del derecho a la ciudad en las últimas décadas se ve atravesado por el debate acerca de las posibilidades y límites que su ejercicio produce, ya que ha sido retomado no solo por movimientos sociales, sino también por organismos de cooperación internacional y por el Estado en todos sus niveles territoriales. Así, las diferentes enunciaciones del derecho a la ciudad, movilizadas por una multiplicidad de actores y movimientos que buscan enmarcar y dar sentido a una serie heterogénea de intervenciones y prácticas urbanas, contienen en su seno intencionalidades y posicionamientos políticos antagónicos. En torno al derecho a la ciudad se dirime una disputa por su significación que, lejos de quedar limitada a un mero arreglo discursivo, se despliega en formas opuestas de entender la participación, la producción del espacio urbano y la capacidad para experimentar arreglos socio-materiales más justos para habitar la ciudad (Minuchin, 2015).

La publicación y circulación de la obra de Lefebvre (1968) se produce en un contexto donde países de la región terminan por consolidar una transición en sus modelos de acumulación, que reposicionan a la ciudad como nodo central de flujos de excedente de capitales y mano de obra.

A diferencia de la apropiación europea, en distintos países de Latinoamérica durante la década del 80 el concepto de derecho a la ciudad permitió la construcción de un marco interpretativo de nuevas modalidades de acción colectiva y acuerdos programáticos para una multiplicidad de actores de la sociedad civil que utilizaron el territorio y la ciudad como una plataforma para proyectar sus proclamas sociales (Cravino, 2009) en un contexto de transiciones democráticas caracterizadas por la creciente precarización de las condiciones físicas y materiales de las ciudades producto de 
los primeros procesos de neoliberalización, por lo general conducidos por dictaduras. La lucha por la ciudad se encuentra con la teoría y le exige un posicionamiento político. Aunque sus reivindicaciones y demandas, objetivamente, no eran anticapitalistas, surgían de nuevos sectores urbanos empobrecidos. Es decir, de aquellos que tenían que resolver con sus propios medios sus necesidades frente a un Estado garante del statu quo de la propiedad urbana (Pradilla Cobos, 2013).

El trabajo y accionar de los distintos colectivos y movimientos traslada a la práctica concreta parte de algunas consideraciones teóricas esgrimidas por Lefebvre en El derecho a la ciudad. Particularmente, en lo referido a la autogestión y el llamado a ampliar el momento de la participación comunitaria en la producción del espacio urbano. La expansión de dichas prácticas territoriales por parte de movimientos sociales urbanos durante la década del '80 y principios de los '90, acumuló conquistas - como el reconocimiento a la posesión y ocupación de tierras - y retrocesos en cuanto a los impactos e injusticias derivadas de procesos de gentrificación vinculados con grandes proyectos urbanos (Cuenya, 2009)

Sin embargo, al mismo tiempo que se consolidaban nuevos repertorios de activismo urbano que encontraban en la construcción, el cooperativismo y la horizontalidad nuevos medios para pensar y hacer política urbana, una serie de innovaciones normativas buscaron institucionalizar el concepto del derecho a la ciudad a lo largo de la región.

La apropiación y traslación normativa del derecho a la ciudad, ampara y legitima institucionalmente al conjunto de reclamos sociales, reconociendo la centralidad del conflicto propiciado por la dinámica del suelo en las ciudades, el derecho a la vivienda digna y, más importante aún, la incorporación a la administración de las plusvalías generadas por procesos de regeneración urbana y a la participación en la producción social del espacio. Se acentúa este último aspecto, ya que permite identificar la expansión de la capacidad fiscal de los gobiernos locales como su incidencia en la producción de suelo urbano mediante el planeamiento estratégico.

Se identifican una serie de acontecimientos políticos que materializan la apropiación normativa del derecho a la ciudad. Entre ellos la instrumentación de la Ley 388 en Colombia sobre la recuperación de plusvalías urbanas, la redacción del Estatuto de las Ciudades en Brasil en 2001 y la nueva Constitución de la República de Ecuador (2008) y su respectivo código de ordenamiento territorial. A escala continental y mundial, se destaca la redacción de la Carta Mundial sobre el Derecho a la Ciudad, producida en Quito en 2004 y luego ratificada en Barcelona con el apoyo de Naciones Unidas y el Foro Urbano Mundial. Es posible afirmar, que la promoción del derecho a la ciudad y el consecuente impulso de políticas públicas urbanas para ciudades 'más justas, sustentables y democráticas', cimentó una alianza de principios entre municipios, agencias públicas nacionales y organismos internacionales (Minuchin, 2015).

La tensión a la que se hace referencia es visible en la contradicción entre la acumulación de instrumentos institucionales que permiten atender las consecuencias sociales y físicas de un proceso de urbanización desigual como el que caracterizó 
a las ciudades latinoamericanas y las cíclicas crisis fiscales que llevaron a gobiernos municipales a habilitar y, en muchos casos, a propiciar la diseminación de una serie de intervenciones urbanas - como tipologías exclusivas, (barrios cerrados, clubes de campo) y grandes proyectos urbanos (Cuenya, 2009) - que lejos de contrarrestar procesos de injusticia espacial, tendieron a multiplicarlos y acentuarlos. Borja (2013) cita el ejemplo del Plan Estratégico como una herramienta contradictoria que, según a que actores considere relevantes, puede favorecer tanto una asociación de cúpulas políticas y económicas como procesos participativos, y puede convertirse en un proyecto político transformador de las ciudades o legitimar las prácticas tradicionales que producen las desigualdades actuales.

Se hace referencia a un proceso de descomposición institucional donde los municipios, atrapados por los problemas presupuestarios, reducen sus servicios generando un amplio margen de acción a inversores privados, empresas locales y regionales, y especuladores inmobiliarios. Esta reforma del Estado que pretende promover la transparencia, el diálogo con los ciudadanos y una mayor participación local en la elaboración de políticas públicas y administración de servicios es el principal factor que afecta actualmente las oportunidades políticas (Grimson, Roberts y Portes, 2005). En este escenario, tanto la apropiación normativa-estatal, como el empoderamiento de gobiernos locales, en general se mostraron incapaces de iniciar procesos de reformas estructurales cercanas a lo proyectado por Lefebvre en el derecho a la ciudad que analizaremos a continuación.

\section{Derecho a la ciudad y bienes comunes}

Interesa retomar el planteo original de Lefebvre sobre el derecho a la ciudad con el propósito de analizarlo en la actualidad como objeto de disputa en el sur global. Entendiendo, de acuerdo a Boaventura de Sousa Santos (2011), que no existe una divisoria lineal entre el sur y el norte global, sino que el escenario de disputa es mucho más complejo porque en el norte global también hay un sur global y viceversa; y en cada uno de estos espacios las tensiones en conflicto se expresan tanto material como simbólicamente, en el marco de la disonancia entre la teoría crítica socio-política y las múltiples prácticas políticas, económicas, sociales y culturales realmente existentes. Por ello el autor propone construir las epistemologías del sur, como un pensamiento focalizado en las alternativas, lo cual invita a plantear la consideración del derecho a la ciudad como objeto de disputa, para aportar al pensamiento crítico latinoamericano sobre la problemática urbana, más allá de su ubicación geográfica.

Desde esta mirada interesa abordar tres ejes de análisis. En primer lugar, las características de los contextos políticos, económicos, sociales y culturales de ambos momentos. Por una parte, el de la ciudad de París en la década de 1960, lugar donde Lefebvre escribe su ensayo sobre el derecho 
a la ciudad, y por otra, el de las ciudades del sur global en las últimas décadas. En segundo lugar, se propone focalizar sobre los actores sociales implicados, los llamados a liderar los procesos revolucionarios o reformistas, por un lado, y el Estado en sus distintos niveles, por otro. Finalmente, el tercer eje se refiere a los bienes comunes urbanos.

Lefebvre escribe El derecho a la ciudad en el año 1967. Éste se plantea, al mismo tiempo, como una queja y una reivindicación. La queja responde al dolor existencial ante lo que él considera la crisis agónica en la vida cotidiana de su ciudad, París. La reivindicación es algo más compleja, pues exige mirar de frente tal crisis y crear una vida urbana alternativa menos alienada, más significativa y gozosa, aunque abierta al futuro, a los encuentros y a la búsqueda perpetua de la novedad (Harvey, 2013).

Ahora bien, ¿a qué crisis agónica se refería Lefebvre en la ciudad de París en 1967? Nada menos que a la progresiva transformación material y simbólica de la ciudad mediante la construcción de vías rápidas, edificios en altura y conjuntos habitacionales de vivienda social, tanto como a la difusión de nuevas pautas de consumo en sus calles y tiendas; lo cual codujo a la desintegración de lo que en otro tiempo había sido una vibrante vida vecinal, así como a la degradación de la fantástica arquitectura de ciertos barrios parisinos. En lo referido a los actores implicados en la construcción del derecho a la ciudad, aquellos supuestamente posicionados para conducir (y construir) dichos procesos, Lefebvre invoca a "la clase obrera", no exclusivamente el proletariado basado en las fábricas, sino a trabajadores urbanos de muy diversos tipos que constituyen una formación de clase muy diferente: fragmentados y divididos, múltiples en sus deseos y necesidades, muy a menudo itinerantes, desorganizados y fluidos mas que sólidamente implantados. ${ }^{1}$

En coincidencia con estas afirmaciones, Harvey (2013), sostiene que a pesar de que a gran parte de la izquierda tradicional le resulta difícil captar el potencial revolucionario de los movimientos sociales urbanos, en gran parte del mundo capitalista avanzado las fábricas han disminuido considerablemente, diezmando la clase obrera industrial clásica. Consecuentemente reconoce el creciente protagonismo de trabajadores eventuales, a menudo a tiempo parcial, desorganizados y mal pagados. En sus palabras, el llamado "precariado" ha desplazado al "proletariado" tradicional y desde su perspectiva, de haber algún movimiento revolucionario en nuestra época, será el "precariado" quien lo realice. Por otro lado, Pradilla Cobos (2013) destaca el paso de las luchas populares por demandas propias de los pobres a temas pluriclasistas, lo que no significa que no existan conflictos de clase subyacentes, en los que los sujetos sociales actúan como ciudadanos en el marco de las libertades individuales. En muchas ciudades del continente los protagonistas son ahora asociaciones de vecinos que defienden el entorno construido (el barrio, la calle) y su calidad de vida de las acciones públicas y privadas (o la combinación de ambas).

Por lo tanto, se considera oportuno diferenciar aquellos movimientos sociales que usan la ciudad como escenario de reivindicaciones particulares, de aquellos otros para los que la ciudad es el objeto de disputa 
y materia para la producción de políticas publicas no estatales. En tal sentido, en el nuevo contexto social y político de la última década, comienza a verificarse el surgimiento de movimientos sociales urbanos, a los que podríamos Ilamar sujetos emergentes, que resisten la expulsión de determinados sectores de la vida urbana, a la vez que reclaman por el acceso igualitario a la misma y actúan en pos de lograr formas de urbanización y de uso del suelo contrapuestos a las dominantes (Schiavo, Vera y Santos Nogueira, 2013). En suma, se entiende que los movimientos sociales urbanos centrados en la ciudad como objeto de disputa constituyen actores clave para pensar y promover el derecho a la ciudad en la región.

Finalmente, interesa resaltar el concepto de bienes comunes y particularmente el de bienes comunes urbanos, en los términos planteados por García Linera (2012) y Harvey (2013), en tanto se considera que la urbanización latinoamericana en el capitalismo actual, volviendo a la mirada de Natanson (2014), necesita crecer puertas afuera. Entonces, son los bienes comunes urbanos una de las claves para avanzar tanto en el pensamiento crítico, como iniciativa autónoma del pensamiento hegemónico, y también en la construcción y materialización del derecho a la ciudad. Por todo esto es que García Linera afirma que el pos-neoliberalismo aún se desenvuelve en los márgenes del régimen del capitalismo, pero contiene, de forma latente, un potencial pos-capitalista.

La clave es un cambio de paradigma en la gestión de lo común. Para que el posneoliberalismo dé el paso hacia un tipo de poscapitalismo se debe avanzar, principalmente, en el esquema de la conversión de la propiedad estatal en propiedad pública y luego en propiedad comunal local-universal (García Linera, 2012). Es decir, transformar los bienes públicos en bienes comunes. Esto se traduce en que la propiedad del Estado no solamente esté bajo el control de los administradores del Estado sino, gradualmente, bajo el control de la propia sociedad. Pero si hablamos de transición, también debemos poner en el centro la democratización del Estado como impulso fundamental para la socialización de la propiedad y la administración de los bienes comunes.

En este contexto es donde cobra importancia el resurgimiento de una retórica y una teoría de los bienes comunes. La participación de los habitantes en la planeación y la gestión de sus territorios, la erradicación de la violencia, la sustentabilidad ambiental, la integración socio-territorial. En definitiva, la garantía y exigibilidad del derecho a la ciudad.

\section{Conclusiones}

En este trabajo se realizó una primera apuesta a la construcción de una perspectiva latinoamericana, o del sur, en términos de las nuevas epistemologías que propone Boaventura de Sousa Santos. El derecho a la ciudad, en los últimos años, viene adquiriendo relevancia tanto para movimientos sociales, agrupaciones políticas de diversas ideologías, gobiernos de distintos estamentos y organismos internacionales. La alusión y el uso permanente del término lejos de aclarar su definición la hace cada vez más flexible 
y borrosa, dando lugar a interpretaciones múltiples y contradictorias.

En este sentido, con el objetivo de clarificar algunos aspectos de lo que se considera "derecho a la ciudad" desde una perspectiva latinoamericana, se eligió como estrategia reflexionar sobre dos tensiones que se consideran claves para el replanteo y el despliegue del derecho a la ciudad.

En la primera tensión se abordaron dos modelos de sociedad determinados por el neoliberalismo y el pos-neoliberalismo. Si el neoliberalismo implicó la retracción del Estado y la acumulación privada de la riqueza; el posneoliberalismo logró poner al Estado en el centro nuevamente abriendo posibilidades de diálogo entre diferentes actores en pos de configurar algunas líneas de trabajo sobre el derecho a la ciudad. De todos modos estos procesos fueron disímiles en la región.

Esta tensión lejos de estar resuelta afronta actualmente una nueva torción a nivel regional $y$, seguramente, la lucha por el derecho a la ciudad y la gestión de los bienes comunes se acentuará ante el visible avance de los intereses privados sobre las propiedades estatales y/o públicas.

La segunda tensión analizada se relaciona con la anterior, pero pone el foco en qué tipo de construcción respecto del derecho a la ciudad se realizó por parte de los gobiernos pos-neoliberales. Aquí se pudo comprobar que si bien el térmico logró instalarse en las agendas de políticas públicas, no se ha superado la etapa normativa. Es decir, en la mayoría de los casos funcionó como argumento de ciertas contemplaciones normativas institucionales pero no se ha emprendido una verdadera transformación política que dispute los sentidos y la materialidad que la matriz neoliberal imprimió en las ciudades latinoamericanas.

En la actualidad, en América Latina, las carencias y límites para la construcción del derecho a la ciudad, al igual que en el contexto de la formulación lefebvriana, no se refieren necesariamente a la recuperación de un modelo de ciudad ideal, en tanto y en cuanto dicho modelo, en la actualidad, continúa siendo parte de la experiencia o la representación simbólica de escasos sectores de la población, a pesar de los avances realizados en la última década en tal sentido.

El avance por la construcción de una perspectiva alternativa del derecho a la ciudad en la coyuntura actual radica en la lucha por los bienes comunes. Será un desafío para los distintos actores implicados en esta problemática elaborar nuevos modos para desplegar esos procesos. En definitiva, lo alternativo, no son los bienes en sí mismos, sino las estrategias y mecanismos, los modos de luchar, legitimar y transformar la realidad urbana contemporánea. 


\section{Ester Schiavo}

Universidad Nacional de Quilmes, Departamento de Ciencias Sociales. Buenos Aires, Argentina. eschiavo@unq.edu.ar

\section{Alejandro Gelfuso}

Universidad Nacional de Rosario, Facultad de Ciencia Política y Relaciones Internacionales. Rosario, Santa Fe, Argentina alegelfuso@gmail.com

\section{Paula Vera}

Consejo Nacional de Investigaciones Científicas y Técnicas, Centro de Estudios sobre Ciencia, Desarrollo y Educación Superior. Ciudad de Buenos Aires, Argentina. paulavera.arg@gmail.com

\section{Notas}

(*) Una versión preliminar de este artículo fue presentada en el I Congreso Latinoamericano de Teoría Social desarrollado en Buenos Aires, del 19 al 21 de agosto de 2015.

(1) Tesis compartida en obras posteriores de la sociología urbana, particularmente en las de un antiguo discípulo de Lefebvre, Manuel Castells, al hacer referencia a los movimientos sociales urbanos.

\section{Referencias}

BAREMBOIN, C. (2010). Transformaciones urbanas en las ciudades latinoamericanas contemporáneas. En: VIII BIENAL DEL COLOQUIO DE TRANSFORMACIONES TERRITORIALES. Anales. Buenos Aires, Argentina

BORJA, J. (2013). Revolución urbana y derechos ciudadanos. Buenos Aires, Ediciones Café de las Ciudades.

CRAVINO, M. A. (2009). Entre el arraigo y el desalojo. La villa 31 de Retiro. Derecho a la ciudad, capital inmobiliario y gestión urbana. Buenos Aires, Universidad Nacional de General Sarmiento.

CUENYA, B. (2009). Grandes proyectos urbanos latinoamericanos. Aportes para su conceptualización desde la perspectiva del gobierno local. Cuaderno Urbano, n. 8, pp. 229-252.

DE SOUSA SANTOS, B. (2011). Una epistemología del sur. Buenos Aires, Siglo XXI.

GAGO, V. (2014). La razón neoliberal. Economías barrocas y pragmática popular. Buenos Aires, Tinta Limón. 
GARCÍA LINERA, A. (2012). Propiedad privada, propiedad pública y comunidad. En: VI ENCUENTRO INTERNACIONAL DE ECONOMÍA POLÍTICA Y DERECHOS HUMANOS. Anales. Buenos Aires, Argentina, Centro de Estudios Económicos y Monitores de las Políticas Públicas - Universidad de las Madres de Plaza de Mayo.

GORELIK, A. (2004). Miradas sobre Buenos Aires. Historia cultural y crítica urbana. Buenos Aires, Siglo XXI.

GRIMSON, A.; ROBERTS, B. y PORTES, A. (2005). Ciudades Latinoamericanas. Un análisis comparativo en el umbral del nuevo siglo. Buenos Aires, Prometeo.

HARVEY, D. (2012). Espacios de esperanza. Madrid, Ediciones AKAL

(2013). Ciudades rebeldes. Del derecho a la ciudad a la revolución urbana. Madrid, Ediciones Akal.

LACLAU, E. (2005). La razón populista. Buenos Aires, Fondo de Cultura Económica de Argentina

LEFEBVRE, H. (1969). El derecho a la ciudad. Barcelona, Península.

MINUCHIN, (2015). Autogestión, articulación y construcción: historia, teoría y práctica del derecho a la ciudad en América Latina. Manchester, University of Manchester.

MONGIN, O. (2004). La condición urbana. La ciudad a la hora de la mundialización. Buenos Aires, Paidos.

NATANSON, J. (2014). Nuestro ogro filantrópico. Le Monde Diplomatique, n. 179. Buenos Aires.

PRADILLA COBOS, E. (2013). “Empresarios, gobiernos y ciudadanos en la disputa por la ciudad”. En: RIBEIRO, A. C. T. et al. Politica giovernamental e ação social no espaço. Rio de Janeiro, Anpur.

SADER, E. (2008). Refundar el Estado. Posneoliberalismo en América Latina. Buenos Aires, Argentina, Ediciones CTA - Consejo Latinoamericano de Ciencias Sociales (Clacso).

SCHIAVO, E.; VERA, P. y SANTOS NOGUEIRA, C. dos (2013). Los movimientos sociales y formas de urbanización metropolitana en el marco del posneoliberalismo. El caso de Giros en la ciudad de Rosario. Revista Quid 16, n. 3, pp. 157-169. Disponible en: http://revistasiigg.sociales.uba.ar/ index.php/quid16/article/view/415/366.

THEODORE, N.; BRENNER, N. y PECK, J. (2009). Urbanismo neoliberal: la ciudad y el imperio de los mercados. Temas Sociales. Santiago, n. 66, pp. 1-11.

Texto recebido em 19/jul/2016

Texto aprovado em 14/nov/2016 